\title{
Acoustic characteristics of voice and speech in Arabic-speaking stuttering children
}

\author{
Marwa Elsherbeny ${ }^{1}$, Hemmat Baz² and Omayma Afsah² ${ }^{2 *}$
}

\begin{abstract}
Background: Using different methodologies, several researchers have reported certain acoustical and physiological differences between fluent utterances of stutterers and normally fluent speakers. The aim of this study was to determine acoustic characteristics of voice and speech in Arabic-speaking stuttering children in comparison to normal children and correlate these characteristics with stuttering severity. A sample of 80 Arabic-speaking Egyptian children (including 40 typically developing children and 40 stuttering children) in the age range 5-8 years were subjected to acoustic analysis of voice and speech using the Praat software.

Results: The stuttering children showed significantly higher values of jitter and shimmer in prolonged /a/ vowel sample, as compared to the normal group. This may reflect the subtle differences in laryngeal functioning or in the complex interaction among the laryngeal, respiratory, and the vocal tract systems in stuttering children. Both jitter and shimmer of prolonged /a/ vowel demonstrated significant positive moderate correlation with stuttering severity as assessed by SSI3. FO was significantly higher in females than in males, both in normal and stuttering children.

Conclusions: The present study revealed significant differences in the acoustic parameters of voice and speech between Arabic-speaking stuttering children and normal children. Some of these acoustic parameters were significantly correlated with stuttering severity. Acoustic analysis can be used as simple, quick, and cheap tool for assessment of stuttering in children and might be a valuable addition to the diagnostic set for assessment of stuttering severity.
\end{abstract}

Keywords: Acoustics, Speech, Stuttering

\section{Background}

Evidence for differences in speech motor control in the stuttering population has been documented in behavioral [1] and neurological [2] paradigms. During stuttering there is abnormal functioning of the whole speech system including the larynx. Abnormal functioning of the larynx may include excessive muscular tension and variable subglottal pressure, which could be caused by muscle incoordination of the respiratory tract. Weaker laryngeal neuromuscular control and disturbances in respiratory and laryngeal control may also lead to voice problems [3].

\footnotetext{
*Correspondence: omafsah@yahoo.com

2 Phoniatric Unit, ORL Department, Faculty of Medicine, Mansoura

University, Mansoura 35516, Egypt

Full list of author information is available at the end of the article
}

Acoustic analysis can determine the laryngeal and supra-laryngeal articulatory behavior of persons who stutter [4]. Data on various aspects of laryngeal function in stuttering children may enhance understanding of this speech disorder [5]. The Praat software program is a tool for phonetic speech analysis. It was developed by Paul Boersma and David Weenink in the Institute of Phonetic Sciences of the University of Amsterdam [6]. The Praat program is free, so it is available for all the voice professionals, in institutions or private offices [7]. It offers packages for the most common computer operating systems (e.g., Windows, Macintosh, Linux) and can therefore be applied regardless operating platforms used by the clinician [8]. The program was utilized for voice and speech analysis in many published studies such as Juste et al. [9], Hasseltineet al [10]., and Rezai et al. [11].

\section{Springer Open}

(c) The Author(s) 2021. Open Access This article is licensed under a Creative Commons Attribution 4.0 International License, which permits use, sharing, adaptation, distribution and reproduction in any medium or format, as long as you give appropriate credit to the original author(s) and the source, provide a link to the Creative Commons licence, and indicate if changes were made. The images or other third party material in this article are included in the article's Creative Commons licence, unless indicated otherwise in a credit line to the material. If material is not included in the article's Creative Commons licence and your intended use is not permitted by statutory regulation or exceeds the permitted use, you will need to obtain permission directly from the copyright holder. To view a copy of this licence, visit http://creativecommons.org/licenses/by/4.0/. 
Speech acoustics of children who stutter (CWS) has been widely investigated. Many researchers have focused on the temporal (voice onset time (VOT), vowel duration, consonant closure time, etc.) and spectral (formant frequencies) aspects of stuttered events [12-14]. Using different methodologies, several studies reported certain acoustical and physiological differences between perceptually fluent utterances of stutterers and normally fluent speakers [5]. The majority of studies addressing laryngeal functioning in stutterers focused on adults and essentially analyzed voice samples through prolonged vowels. Studies conducted on children primarily analyzed prolonged vowels or spontaneous speech samples. The aim of this study was to determine acoustic characteristics of voice and speech in Arabic-speaking stuttering children in comparison to normal children and correlate these characteristics with stuttering severity. The use of both voice and speech samples, including automatic as well as spontaneous speech samples, would allow for better understanding of laryngeal dynamics in stutterers and might highlight the role of acoustic analysis in follow up of these children.

\section{Methods}

The present study was an observational analytical casecontrol study. It was conducted on 80 Arabic-speaking Egyptian children (including 40 typically developing children (group I) and 40 stuttering children (group II) in the age range 5-8 years in the period from March 2019 to March 2021. Group I children were collected from schools or from the relatives of children presenting to Phoniatric outpatient clinic, and group II children were selected from children presented to Phoniatric outpatient clinic by convenience sampling.

Group I children included typically developing children who displayed age-appropriate normal speech and language skills, with no history of voice disorders. Exclusion criteria for group II were children with hearing impairment (as the acoustic parameters in children with hearing impairment differ from those in normal children), children with neurological disorders, such as brain damaged motorly handicapped children (BDMH) (as acoustic parameters can be affected by the central nervous system pathology), children with other speech disorders, children with language or voice disorders, and children with history of previous speech therapy.

Written informed consent was obtained from parents of children participating in the study. The study was approved by the Institutional Research Board of Faculty of Medicine (MS.19.03.523).

Children in group II (stuttering children) were subjected to full history taking, full general examination, and subjective evaluation of language, speech, and voice to exclude children with other speech disorders as well as dysphonic children. Auditory perceptual assessment (APA) of speech was used as subjective tool for evaluation of the child's speech (both automatic and spontaneous speech) to determine the pattern of dysfluencies. General examination and vocal tract examination were done to rule out health problems that affect speech development. Assessment of stuttering severity was done using stuttering severity instrument "SSI3" [15] and Bloodstien classification [16].

Children in both study groups were subjected to computerized acoustic analysis of their speech and voice, using the Praat software version 6.0.36 [17]. Analysis was done on two speech samples and sustained vowel /a/, with elimination of the irregularities in the beginning and end of utterance. The recording time in the Praat software was adjusted to fit the duration of speech samples. The child was asked to speak at "comfortable loudness and pitch." The two speech samples included:

a) Automatic speech: counting from one to ten.

b) Spontaneous speech.

The recording process was done for each child individually by using unidirectional microphone (bm7000-usb) in a quiet room. The distance between the microphone and the mouth of the child was about $15 \mathrm{~cm}$. All the recorded sounds were saved onto a personal computer as separate wave files.

The following parameters were computed with Praat: fundamental frequency (fo) (in HZ), jitter percent, shimmer percent, and harmonic to noise ratio (in $\mathrm{dB}$ ).

Data were coded, computed, and then analyzed using IBM SPSS (Statistical package for social science) version 24 for Windows. Qualitative data were presented by frequency tables (number and percentages). For quantitative variables, the normality of data was first tested with Shapiro-Wilk test. Then, the data were presented by central indices and dispersion: mean \pm standard deviation (SD) for normally distributed variables and median (minimum-maximum) for non-normally distributed variables.

Chi-square test was used to test association between categorical variables. Association between normally distributed continuous variables was tested using independent sample $t$ test in 2 independent groups, while Mann-Whitney $U$ test $(z)$ was used to compare 2 independent non-normally distributed continuous variables. The one-way analysis of variance (ANOVA) was used to determine whether there were any statistically significant differences between the means of two or more independent (unrelated) groups. Also, Kruskal-Wallis $H$ test was used to compare non-normally distributed continuous variables in more than two different groups. 
Table 1 Demographic characteristics of the studied groups

\begin{tabular}{|c|c|c|c|}
\hline Parameters & $\begin{array}{l}\text { Normal group } \\
n=40\end{array}$ & $\begin{array}{l}\text { Stuttering group } \\
n=40\end{array}$ & $\begin{array}{l}\text { Test of } \\
\text { significance }\end{array}$ \\
\hline \multicolumn{4}{|l|}{ Age } \\
\hline $\begin{array}{l}\text { mean } \pm S D \\
(\min -m a x)\end{array}$ & $\begin{array}{l}6.7 \pm 1.1 \\
(5-8)\end{array}$ & $\begin{array}{l}6.3 \pm 1.1 \\
(5-8)\end{array}$ & $\begin{array}{l}t=1.6 \\
P=1.3\end{array}$ \\
\hline \multicolumn{4}{|l|}{ Gender } \\
\hline $\begin{array}{l}>\text { Male } \\
>\text { Female }\end{array}$ & $\begin{array}{l}20(50 \%) \\
20(50 \%)\end{array}$ & $\begin{array}{l}22(55 \%) \\
18(45 \%)\end{array}$ & $\begin{array}{l}X^{2}=0.20 \\
P=0.65\end{array}$ \\
\hline
\end{tabular}

Data expressed as number (\%) or mean $\pm \mathrm{SD}$. $\chi^{2}$, chi-square test; $t$, independent samples $t$-test; not significant: $p>0.05$

Table 2 Distribution of group || children based on stuttering severity

\begin{tabular}{lll}
\hline Parameters & Degree & $\boldsymbol{N}=\mathbf{4 0}$ \\
\hline SSI3 & $>$ Mild & $17(42.5 \%)$ \\
& $>$ Moderate & $16(40 \%)$ \\
& $>$ Severe & $7(17.5 \%)$ \\
& $\rightarrow$ Mean \pm SD of SSI3 & $22.7 \pm 6.3$ \\
Bloodstien (BLD) & $>$ I & $4(10 \%)$ \\
& $>$ II & $16(40 \%)$ \\
& $>$ III & $16(40 \%)$ \\
& $>$ IV & $4(10 \%)$
\end{tabular}

Data expressed as number (\%) or mean \pm SD

Pearson correlation was used to correlate normally distributed data, while Spearman correlation was used to correlate non-normally distributed data. For all the abovementioned statistical tests, the results were considered significant when the probability of error was less than or equal to $5 \%(p \leq 0.05)$.

\section{Results}

\section{Descriptive data}

\section{Demographic characteristics of the studied groups}

The present study was conducted on a sample of 80 Arabic-speaking Egyptian children in the age range 5-8 years (mean $6.5 \pm 1.1$ years), including 42 males and 38 females, arranged into 2 groups as follows: Group I: composed of 40 typically developing children with mean age $6.7 \pm 1.1$ years. They included 20 males $(50 \%)$ and 20 females (50\%). Group II: composed of 40 stuttering children with mean age $6.3 \pm 1.1$ years. They included 22 males (55\%) and 18 females (45\%). Both groups were matched for age and gender (Table 1).

\section{Distribution of group II children based on stuttering severity}

Based on Bloodstien classification, grades II and III represented the majority of the studied children (40\% each). Based on stuttering severity instrument (SSI3), the majority of children had mild stuttering (42.5\%), followed by moderate (40\%) and severe (17.5\%) degrees (Table 2 ).

\section{Comparative analysis}

Comparison between acoustic parameters in the normal and stuttering groups

In prolonged /a/ vowel sample, the stuttering group showed significantly higher values of jitter and shimmer in comparison to the normal group. The two speech samples showed no significant differences in any of the acoustic parameters between both groups (Table 3 ).

\section{Within group comparison of acoustic parameters across the different samples}

In both normal and stuttering groups, non-significant differences were observed between acoustic parameters of automatic and spontaneous speech samples. On the other hand, jitter and shimmer were significantly lower and harmonic to noise ratio was significantly higher in prolonged /a/ vowel sample in comparison to the two speech samples (Tables 4 and 5).

\section{Associative and correlative analysis Association between acoustic parameters and gender in the normal and stuttering groups}

In all samples, fundamental frequency (F0) was significantly higher in females than in males. On the other hand, neither jitter shimmer nor $\mathrm{H} / \mathrm{N}$ ratio was significantly associated with gender (Table 6).

\section{Correlation between acoustic parameters and SSI3 scores in the stuttering group}

There was significant positive moderate correlation between SSI3 score and both jitter and shimmer of prolonged /a/ vowel. In spontaneous and automatic speech samples, there were non-significant correlations between acoustic parameters and SSI3 scores (Table 7).

\section{Association between acoustic parameters and Bloodstien (BLD) grade in the stuttering group}

None of the acoustic parameters were significantly associated with Bloodstien grade in any of the speech samples (Table 8).

\section{Discussion}

The present study aimed to determine acoustic characteristics of voice and speech in Arabic-speaking stuttering children in comparison to normal children and correlate these characteristics with stuttering severity. A sample of 80 Arabic-speaking Egyptian children (including 40 typically developing children and 40 stuttering children) in the age range 5-8 years were subjected to acoustic analysis of voice and speech using the Praat software.

The inclusion of both speech and sustained vowel in voice analysis was important for several reasons; First, 
Table 3 Acoustic parameters of the normal and stuttering groups

\begin{tabular}{|c|c|c|c|c|}
\hline Parameters & & $\begin{array}{l}\text { Normal group } \\
n=40\end{array}$ & $\begin{array}{l}\text { Stuttering group } \\
n=40\end{array}$ & Test of significance \\
\hline \multirow[t]{4}{*}{ I- Prolonged /a/ vowel } & Fundamental Frequency (F0) & $\begin{array}{l}269.3 \pm 36.8 \\
(210-358.7)\end{array}$ & $\begin{array}{l}272.1 \pm 37.6 \\
(207.8-362.8)\end{array}$ & $\begin{array}{l}t=0.35 \\
P=0.73\end{array}$ \\
\hline & Jitter & $\begin{array}{l}0.43 \\
(0.2-0.82)\end{array}$ & $\begin{array}{l}0.51 \\
(0.23-2.4)\end{array}$ & $\begin{array}{l}Z=2.4 \\
P=\mathbf{0 . 0 2}\end{array}$ \\
\hline & Shimmer & $\begin{array}{l}4.5 \\
(2.6-8.1)\end{array}$ & $\begin{array}{l}5.3 \\
(1.3-12.1)\end{array}$ & $\begin{array}{l}Z=2.1 \\
P=\mathbf{0 . 0 4}\end{array}$ \\
\hline & Harmonic to Noise ratio (H/N ratio) & $\begin{array}{l}18.9 \pm 2.4 \\
(15.2-25.7)\end{array}$ & $\begin{array}{l}17.6 \pm 4.0 \\
(8.9-25.0)\end{array}$ & $\begin{array}{l}t=1.8 \\
P=0.08\end{array}$ \\
\hline \multirow[t]{4}{*}{ II- Automatic speech } & Fundamental Frequency (F0) & $\begin{array}{l}260.3 \pm 33.9 \\
(195.3-373.3)\end{array}$ & $\begin{array}{l}256.2 \pm 37.3 \\
(176.4-336.1)\end{array}$ & $\begin{array}{l}t=0.51 \\
P=0.61\end{array}$ \\
\hline & Jitter & $\begin{array}{l}1.6 \pm 0.5 \\
(0.98-2.9)\end{array}$ & $\begin{array}{l}1.6 \pm 0.5 \\
(0.8-2.6)\end{array}$ & $\begin{array}{l}t=0.41 \\
P=0.69\end{array}$ \\
\hline & Shimmer & $\begin{array}{l}8.0 \pm 1.8 \\
(4.8-12.4)\end{array}$ & $\begin{array}{l}8.7 \pm 2.7 \\
(4.7-12.9)\end{array}$ & $\begin{array}{l}t=1.3 \\
P=0.21\end{array}$ \\
\hline & Harmonic to Noise ratio (H/N ratio) & $\begin{array}{l}13.1 \pm 1.8 \\
(9.9-17.4)\end{array}$ & $\begin{array}{l}13.1 \pm 3.0 \\
(8.4-19.9)\end{array}$ & $\begin{array}{l}t=0.08 \\
P=0.94\end{array}$ \\
\hline \multirow[t]{4}{*}{ III- Spontaneous speech } & Fundamental Frequency (F0) & $\begin{array}{l}276.1 \pm 33.3 \\
(205.7-365.2)\end{array}$ & $\begin{array}{l}276.4 \pm 45.4 \\
(158.0-336.1)\end{array}$ & $\begin{array}{l}t=0.04 \\
P=0.97\end{array}$ \\
\hline & Jitter & $\begin{array}{l}1.7 \pm 0.5 \\
(1.0-3.0)\end{array}$ & $\begin{array}{l}1.7 \pm 0.4 \\
(0.74-2.9)\end{array}$ & $\begin{array}{l}t=0.06 \\
P=0.95\end{array}$ \\
\hline & Shimmer & $\begin{array}{l}8.2 \\
(4.6-14.9)\end{array}$ & $\begin{array}{l}8.2 \\
(1.6-14.8)\end{array}$ & $\begin{array}{l}Z=0.17 \\
P=0.86\end{array}$ \\
\hline & Harmonic to Noise ratio (H/N ratio) & $\begin{array}{l}13.6 \pm 2.2 \\
(10.1-18.0)\end{array}$ & $\begin{array}{l}13.8 \pm 3.0 \\
(8.5-19.7)\end{array}$ & $\begin{array}{l}t=0.40 \\
P=0.69\end{array}$ \\
\hline
\end{tabular}

Data expressed as mean \pm SD or median (minimum-maximum)

*Significant $p \leq 0.05$

$t$, independent samples $t$-test

Z, Mann-Whitney test

Table 4 Within group comparison of acoustic parameters across the different samples

\begin{tabular}{|c|c|c|c|c|c|}
\hline Items & & Prolonged /a/ vowel & Automatic speech & Spontaneous speech & Test of significance \\
\hline \multirow[t]{4}{*}{ Normal group } & Fundamental Frequency (F0) & $\begin{array}{l}269.3 \pm 36.8 \\
(210-358.7)\end{array}$ & $\begin{array}{l}260.3 \pm 33.9 \\
(195.3-373.3)\end{array}$ & $\begin{array}{l}276.1 \pm 33.3 \\
(205.7-365.2)\end{array}$ & $\begin{array}{l}F=2.1 \\
P=0.13\end{array}$ \\
\hline & Jitter & $\begin{array}{l}0.43 \\
(0.2-0.82)\end{array}$ & $\begin{array}{l}1.6 \\
(0.98-2.2)\end{array}$ & $\begin{array}{l}1.6 \\
(1.0-3.0)\end{array}$ & $\begin{array}{l}\mathrm{KW}=79.9 \\
P \leq \mathbf{0 . 0 0 1 *}\end{array}$ \\
\hline & Shimmer & $\begin{array}{l}4.5 \\
(2.6-8.1)\end{array}$ & $\begin{array}{l}7.5 \\
(4.8-12.4)\end{array}$ & $\begin{array}{l}8.2 \\
(4.6-14.9)\end{array}$ & $\begin{array}{l}\mathrm{KW}=66.9 \\
P \leq \mathbf{0 . 0 0 1 *}\end{array}$ \\
\hline & Harmonic to Noise ratio(H/N ratio) & $\begin{array}{l}18.9 \pm 2.4 \\
(15.2-25.7)\end{array}$ & $\begin{array}{l}13.1 \pm 1.8 \\
(9.9-17.4)\end{array}$ & $\begin{array}{l}13.6 \pm 2.2 \\
(10.1-18.0)\end{array}$ & $\begin{array}{l}\mathrm{F}=92.0 \\
\boldsymbol{P} \leq \mathbf{0 . 0 0 1 *}\end{array}$ \\
\hline \multirow[t]{4}{*}{ Stuttering group } & Fundamental Frequency (F0) & $\begin{array}{l}272.1 \pm 37.6 \\
(207.8-362.8)\end{array}$ & $\begin{array}{l}256.2 \pm 37.3 \\
(176.4-336.1)\end{array}$ & $\begin{array}{l}276.4 \pm 45.4 \\
(158.0-336.1)\end{array}$ & $\begin{array}{l}F=2.8 \\
P=0.06\end{array}$ \\
\hline & Jitter & $\begin{array}{l}0.51 \\
(0.23-2.4)\end{array}$ & $\begin{array}{l}1.6 \\
(0.8-2.6)\end{array}$ & $\begin{array}{l}1.7 \\
(0.74-2.9)\end{array}$ & $\begin{array}{l}\mathrm{KW}=52.5 \\
\boldsymbol{P} \leq \mathbf{0 . 0 0 1}\end{array}$ \\
\hline & Shimmer & $\begin{array}{l}5.3 \\
(1.3-12.1)\end{array}$ & $\begin{array}{l}8.6 \\
(4.7-12.9)\end{array}$ & $\begin{array}{l}8.2 \\
(1.6-14.8)\end{array}$ & $\begin{array}{l}\mathrm{KW}=23.6 \\
\boldsymbol{P} \leq \mathbf{0 . 0 0 1 *}\end{array}$ \\
\hline & Harmonic to Noise ratio & $\begin{array}{l}17.6 \pm 4.0 \\
(8.9-25.0)\end{array}$ & $\begin{array}{l}13.1 \pm 3.0 \\
(8.4-19.9)\end{array}$ & $\begin{array}{l}13.8 \pm 3.0 \\
(8.5-19.7)\end{array}$ & $\begin{array}{l}\mathrm{F}=19.9 \\
\boldsymbol{P} \leq \mathbf{0 . 0 0 1 *}\end{array}$ \\
\hline
\end{tabular}

Data expressed as mean \pm SD or median (minimum-maximum)

*Significant $p \leq 0.05$

$\mathrm{F}$, one-way ANOVA test

KW, Kruskal-Wallis test 
Table 5 P1, P2, and P3 of within group comparison between acoustic parameters in the normal and stuttering groups

\begin{tabular}{|c|c|c|c|c|}
\hline Items & & P1 & P2 & P3 \\
\hline \multirow[t]{3}{*}{ Normal group } & Jitter & $\begin{array}{l}Z=7.6 \\
P \leq \mathbf{0 . 0 0 1 *}\end{array}$ & $\begin{array}{l}Z=7.7 \\
P \leq 0.001 *\end{array}$ & $\begin{array}{l}Z=1.1 \\
P=0.28\end{array}$ \\
\hline & Shimmer & $\begin{array}{l}Z=7.2 \\
P \leq \mathbf{0 . 0 0 1 *}\end{array}$ & $\begin{array}{l}Z=6.9 \\
P \leq \mathbf{0 . 0 0 1 *}\end{array}$ & $\begin{array}{l}Z=1.2 \\
P=0.24\end{array}$ \\
\hline & Harmonic to Noise ratio & $\leq 0.001 *$ & $\leq 0.001 *$ & 0.31 \\
\hline \multirow[t]{3}{*}{ Stuttering group } & Jitter & $\begin{array}{l}Z=6.3 \\
P \leq \mathbf{0 . 0 0 1 *}\end{array}$ & $\begin{array}{l}Z=6.2 \\
P \leq 0.001 *\end{array}$ & $\begin{array}{l}Z=0.76 \\
P=0.45\end{array}$ \\
\hline & Shimmer & $\begin{array}{l}Z=4.3 \\
P \leq \mathbf{0 . 0 0 1 *}\end{array}$ & $\begin{array}{l}Z=4.1 \\
P \leq \mathbf{0 . 0 0 1 *}\end{array}$ & $\begin{array}{l}Z=0.27 \\
P=0.79\end{array}$ \\
\hline & Harmonic to Noise ratio & $\leq 0.001 *$ & $\leq 0.001 *$ & 0.38 \\
\hline
\end{tabular}

P1, prolonged /a / vowel vs automatic speech; $P 2$, prolonged /a / vowel vs spontaneous speech; $P 3$, automatic speech vs spontaneous speech; $Z$, Mann-Whitney test Significant difference between groups regarding harmonic to noise ratio is done by post hoc LSD test *Significant $p \leq 0.05$

Table 6 Association between acoustic parameters and gender in the normal and stuttering groups

\begin{tabular}{|c|c|c|c|c|c|c|}
\hline \multirow{2}{*}{$\begin{array}{l}\text { Parameters } \\
\text { Normal group }\end{array}$} & \multirow[b]{2}{*}{ 1- Prolonged /a/ vowel } & \multirow[b]{2}{*}{ F0 } & \multirow{2}{*}{$\begin{array}{l}\begin{array}{l}\text { Male } \\
\boldsymbol{n}=\mathbf{2 2}\end{array} \\
251.2 \pm 33.1\end{array}$} & \multirow{2}{*}{$\begin{array}{l}\begin{array}{l}\text { Female } \\
\boldsymbol{n}=\mathbf{1 8}\end{array} \\
287.5 \pm 31.4\end{array}$} & \multicolumn{2}{|c|}{ Test of significance } \\
\hline & & & & & $t=3.6$ & $P=\mathbf{0 . 0 0 1 *}$ \\
\hline & & Jitter & $0.46(0.2-0.82)$ & $0.37(0.2-0.62)$ & $Z=1.2$ & $P=0.22$ \\
\hline & & Shimmer & $4.4(2.6-6.8)$ & $4.5(2.9-8.1)$ & $Z=0.24$ & $P=0.81$ \\
\hline & & $\mathrm{H} / \mathrm{N}$ ratio & $18.7 \pm 2.5$ & $19.1 \pm 2.2$ & $t=0.45$ & $P=0.63$ \\
\hline & 2- Automatic speech & FO & $249.5 \pm 40.2$ & $271.1 \pm 22.2$ & $t=2.1$ & $P=\mathbf{0 . 0 4} *$ \\
\hline & & Jitter & $1.6 \pm 0.5$ & $1.6 \pm 0.4$ & $t=0.28$ & $P=0.78$ \\
\hline & & Shimmer & $8.2 \pm 1.9$ & $7.9 \pm 1.5$ & $t=0.52$ & $P=0.61$ \\
\hline & & $\mathrm{H} / \mathrm{N}$ ratio & $12.7 \pm 1.7$ & $13.5 \pm 1.8$ & $t=1.5$ & $P=0.13$ \\
\hline & 3- Spontaneous speech & FO & $257.8 \pm 25.4$ & $294.3 \pm 30.5$ & $t=4.1$ & $P \leq \mathbf{0 . 0 0 1 *}$ \\
\hline & & Jitter & $1.7 \pm 0.5$ & $1.6 \pm 0.4$ & $t=0.67$ & $P=0.51$ \\
\hline & & Shimmer & $8.5(4.6-14.9)$ & $8.2(5.3-12.8)$ & $Z=0.08$ & $P=0.94$ \\
\hline & & $\mathrm{H} / \mathrm{N}$ ratio & $13.3 \pm 2.5$ & $13.9 \pm 1.8$ & $t=0.81$ & $P=0.42$ \\
\hline \multirow[t]{12}{*}{ Stuttering group } & 1- Prolonged/a/ vowel & FO & $253.6 \pm 32.4$ & $294.9 \pm 30.8$ & $t=4.1$ & $P \leq \mathbf{0 . 0 0 1 *}$ \\
\hline & & Jitter & $0.49(0.3-2.1)$ & $0.54(0.23-2.4)$ & $Z=0.08$ & $P=0.94$ \\
\hline & & Shimmer & $4.7(1.3-12.1)$ & $5.7(1.9-10.8)$ & $Z=1.0$ & $P=0.31$ \\
\hline & & $\mathrm{H} / \mathrm{N}$ ratio & $17.9 \pm 4.2$ & $17.1 \pm 3.9$ & $t=0.64$ & $P=0.53$ \\
\hline & 2- Automatic speech & F0 & $239.9 \pm 39.4$ & $276.1 \pm 22.5$ & $t=3.5$ & $P=0.001 *$ \\
\hline & & Jitter & $1.7 \pm 0.5$ & $1.6 \pm 0.5$ & $t=0.81$ & $P=0.42$ \\
\hline & & Shimmer & $8.7 \pm 2.5$ & $8.7 \pm 3.0$ & $t=0.05$ & $P=0.96$ \\
\hline & & $\mathrm{H} / \mathrm{N}$ ratio & $13.1 \pm 2.4$ & $13.2 \pm 3.7$ & $t=0.06$ & $P=0.96$ \\
\hline & 3- Spontaneous speech & FO & $255.9 \pm 48.9$ & $301.6 \pm 23.3$ & $t=3.6$ & $P=0.001^{*}$ \\
\hline & & Jitter & $1.8 \pm 0.5$ & $1.6 \pm 0.4$ & $t=1.5$ & $P=0.14$ \\
\hline & & Shimmer & $8.2(4.7-13.8)$ & $8.3(1.6-14.8)$ & $Z=0.10$ & $P=0.92$ \\
\hline & & $\mathrm{H} / \mathrm{N}$ ratio & $13.5 \pm 3.1$ & $14.2 \pm 2.9$ & $t=0.67$ & $P=0.51$ \\
\hline
\end{tabular}

Data expressed as mean \pm SD or median (minimum-maximum)

*Significant $p \leq 0.05$

$t$, independent samples $t$-test

Z, Mann-Whitney test

vocal inconstancies typically observed in continuous speech rather than in sustained vowels (e.g., voice onset/ offset, prosodic modulations, voice breaks, etc.) can be decisive in auditory-perceptual voice quality evaluation [18]. Second, both types can express different types/ degrees of vocal dysfunction and, consequently, result 
Table 7 Correlation between acoustic parameters and SSI3 in the stuttering group

\begin{tabular}{|c|c|c|c|}
\hline \multirow[t]{2}{*}{ Items } & & \multicolumn{2}{|c|}{ Correlation with SSI3 } \\
\hline & & $r$ & $P$ \\
\hline \multirow[t]{4}{*}{ 1-Prolonged /a/vowel } & Fundamental Frequency (F0) & $-0.18^{a}$ & 0.27 \\
\hline & Jitter & $0.43^{b}$ & $0.006^{*}$ \\
\hline & Shimmer & $0.47^{b}$ & $0.002^{*}$ \\
\hline & Harmonic to Noise ratio ( $\mathrm{H} / \mathrm{N}$ ratio) & $-0.23^{\mathrm{a}}$ & 0.15 \\
\hline \multirow[t]{4}{*}{ 2-Automatic speech } & Fundamental Frequency (F0) & $-0.19^{a}$ & 0.24 \\
\hline & Jitter & $0.05^{\mathrm{a}}$ & 0.77 \\
\hline & Shimmer & $0.31^{\mathrm{a}}$ & 0.06 \\
\hline & Harmonic to Noise ratio ( $\mathrm{H} / \mathrm{N}$ ratio) & $-0.13^{a}$ & 0.44 \\
\hline \multirow[t]{4}{*}{ 3-Spontaneous speech } & Fundamental Frequency (F0) & $-0.23^{a}$ & 0.16 \\
\hline & Jitter & $-0.19^{a}$ & 0.25 \\
\hline & Shimmer & $0.13^{b}$ & 0.43 \\
\hline & Harmonic to Noise ratio ( $\mathrm{H} / \mathrm{N}$ ratio) & $-0.10^{\mathrm{a}}$ & 0.52 \\
\hline
\end{tabular}

a Pearson correlation

${ }^{\text {b }}$ Spearman's correlation

$r$, correlation coefficient

*Significant $p \leq 0.05$

Table 8 Association between acoustic parameters and Bloodstien (BLD) grade in the stuttering group

\begin{tabular}{|c|c|c|c|c|c|c|}
\hline Items & & $\begin{array}{l}\text { Grade I } \\
n=4\end{array}$ & $\begin{array}{l}\text { Grade II } \\
n=16\end{array}$ & $\begin{array}{l}\text { Grade III } \\
n=16\end{array}$ & $\begin{array}{l}\text { Grade IV } \\
n=4\end{array}$ & Test of significance \\
\hline \multirow[t]{4}{*}{ I- Prolonged /a / vowel } & Fo & $295.5 \pm 26.1$ & $274.0 \pm 35.7$ & $267.9 \pm 43.8$ & $258.9 \pm 25.4$ & $\begin{array}{l}F=0.75 \\
P=0.53\end{array}$ \\
\hline & Jitter & $\begin{array}{l}0.39 \\
(0.23-0.55)\end{array}$ & $\begin{array}{l}0.64 \\
(0.25-2.4)\end{array}$ & $\begin{array}{l}0.41 \\
(0.3-2.0)\end{array}$ & $\begin{array}{l}0.58 \\
(0.24-0.89)\end{array}$ & $\begin{array}{l}K W=4.4 \\
P=0.22\end{array}$ \\
\hline & Shimmer & $\begin{array}{l}5.3 \\
(2.8-6.4)\end{array}$ & $\begin{array}{l}5.3 \\
(2.0-12.1)\end{array}$ & $\begin{array}{l}4.8 \\
(1.3-11.8)\end{array}$ & $\begin{array}{l}7.2 \\
(2.0-11.7)\end{array}$ & $\begin{array}{l}K W=0.56 \\
P=0.91\end{array}$ \\
\hline & $\mathrm{H} / \mathrm{N}$ ratio & $18.9 \pm 2.0$ & $16.7 \pm 4.1$ & $18.4 \pm 4.6$ & $16.4 \pm 3.1$ & $\begin{array}{l}F=0.70 \\
P=0.56\end{array}$ \\
\hline \multirow[t]{4}{*}{ II- Automatic speech } & F0 & $282.7 \pm 26.1$ & $257.7 \pm 34.9$ & $249.1 \pm 44.4$ & $251.8 \pm 12.4$ & $\begin{array}{l}F=0.89 \\
P=0.46\end{array}$ \\
\hline & Jitter & $1.7 \pm 0.5$ & $1.7 \pm 0.5$ & $1.5 \pm 0.5$ & $1.9 \pm 0.6$ & $\begin{array}{l}F=0.63 \\
P=0.59\end{array}$ \\
\hline & Shimmer & $8.9 \pm 2.5$ & $8.6 \pm 2.9$ & $8.5 \pm 2.5$ & $9.2 \pm 3.0$ & $\begin{array}{l}F=0.08 \\
P=0.97\end{array}$ \\
\hline & $\mathrm{H} / \mathrm{N}$ ratio & $13.5 \pm 2.6$ & $13.2 \pm 3.6$ & $13.2 \pm 2.5$ & $12.4 \pm 3.8$ & $\begin{array}{l}F=0.11 \\
P=0.96\end{array}$ \\
\hline \multirow[t]{4}{*}{ III-Spontaneous speech } & FO & $299.4 \pm 19.8$ & $279.7 \pm 44.3$ & $268.3 \pm 55.3$ & $273.0 \pm 11.6$ & $\begin{array}{l}F=0.53 \\
P=0.67\end{array}$ \\
\hline & Jitter & $1.8 \pm 0.3$ & $1.6 \pm 0.4$ & $1.7 \pm 0.5$ & $1.7 \pm 0.4$ & $\begin{array}{l}F=0.13 \\
P=0.94\end{array}$ \\
\hline & Shimmer & $\begin{array}{l}10.3 \\
(6.7-14.8)\end{array}$ & $\begin{array}{l}7.3 \\
(5.4-14.5)\end{array}$ & $\begin{array}{l}9.6 \\
(4.7-13.8)\end{array}$ & $\begin{array}{l}4.4 \\
(1.6-11.2)\end{array}$ & $\begin{array}{l}K W=3.5 \\
P=0.32\end{array}$ \\
\hline & $\mathrm{H} / \mathrm{N}$ ratio & $13.6 \pm 3.2$ & $14.3 \pm 3.2$ & $13.4 \pm 3.2$ & $13.7 \pm 2.3$ & $\begin{array}{l}F=0.23 \\
P=0.88\end{array}$ \\
\hline
\end{tabular}

in different perceptual ratings [19]. Adductor spasmodic dysphonia, for example, can often be characterized by relatively normal voice during sustained vowels, whereas voice in continuous speech is often more severely disrupted [20]. Third, dysphonia symptoms commonly emerge in conversational voice production instead of sustained vowels (except for singing voice) and they are usually revealed to patients in connected speech [21]. 
In this study, comparison between acoustic parameters in normal and stuttering groups showed that in prolonged /a/ vowel sample, the stuttering group demonstrated significantly higher values of jitter and shimmer in comparison to the normal group. This finding coincided with previous studies by Bolfan-Stosic and Prizl [22] and Salihovic et al. [3] and indicated that the sustained phonations of the stutterers were less stable than those of the non-stutterers in terms of both vocal frequency and intensity. On the other hand, Gharamaleki et al. [23] argued that there was no significant difference between normal and stuttering children acoustically.

Wertzner et al. [24] suggested that the jitter may be affected mainly because of lack of control of vocal fold vibration at the moments of stuttering which may result in the presence of noise at emission and breathiness of the voice. Baken [25] explained amplitude variation by the fact that the intensity depends on interaction between subglottal pressure and aerodynamics at vocal folds level. Zocchi et al. [26] demonstrated that stuttering individuals have variable, sometimes even chaotic subglottal pressure.

Hall and Yairi [5] stated that shimmer values were substantially higher in stuttering children than in normal children which may indicate that subtle differences in laryngeal functioning or in the complex interaction among the laryngeal, respiratory, and the vocal tract systems are present at very early stages of the disorder.

The present study revealed non-significant difference between stuttering and normal children as regard fundamental frequency (fo). This result is in harmony with that detected by Schmitt and Cooper [27] and Gharamaleki et al. [23]. On the other hand Hall and Yairi [5] concluded that "stuttering children tended to exhibit slightly lower fundamental frequency than normally fluent children." Salihovic et al. [3] claimed that abnormal functioning of the larynx may include excessive muscular tension and variable subglottal pressure, which could be caused by muscle incoordination of the respiratory tract.

Fundamental frequency (f0) is the acoustic correlate of pitch; it is affected by the degree of tension in the larynx as well as by the aerodynamic forces and muscle actions. Variations in pitch level, intonation metrical structure, and phrasing are aspects of prosody which is considered a key element in acquiring and producing meaningful language [28].

Fosnot and Jun [29] investigated intonation and timing characteristics of stuttering children's speech and compared the prosodic characteristics with normal control children both quantitatively (in terms of pitch range and duration) and qualitatively (in terms of the type of pitch accents, boundary tones, and phrasing). The authors reported that stuttering children differed only slightly from normal control subjects regarding most measurements.

Salihovic et al. [3] documented that differences between stuttering and normally fluent speakers in phonation parameters are more pronounced in stuttering adults than in stuttering children and those differences occur as reflection of usual compensatory behavior in reaction to dysfluencies and cannot be considered as etiologic stuttering factor.

In the current study, within group comparison of acoustic parameters across the 3 different samples showed that jitter and shimmer were significantly lower and harmonic to noise ratio was significantly higher in prolonged / a/ vowel as compared to automatic and spontaneous speech samples in both normal and stuttering children. This could be explained by the potential differences in the quality of phonation that exist between the different phonatory samples. As stated by Wolfe et al. [30], the quality of the laryngeal tone in speech samples is subjected to articulatory changes that do not occur during static vowel productions. The production of consonants has more diverse acoustic characteristics than vowels do. Furthermore, voicing is the only source of sound source in vowel production while in consonant voicing is not the only sound as there is coordination between voicing, aspiration (bursts of air), and frication (noise produced when air goes through a constriction) [31].

The present study revealed significantly higher fundamental frequency (F0) in females than males even before puberty in both normal and stuttering groups in the different samples. The obtained results were in line with that detected by Abo-Ras et al. [32], who demonstrated that F0 was lower in males than in females in their study on normal children in the age range 4-12 years. The same authors demonstrated that jitter and shimmer did not differ significantly between males and females.

On the other hand, Toki et al. [33] showed that boys and girls up to the age of 12 years have no significant differences in their mean fundamental frequency as $\mathrm{f} 0$ is directly related to the length, stress, rigidity, and mass of the vocal folds. Fitch and Giedd [34] declared that acoustic differences become more apparent after age 12 where discrete male-female differences in $\mathrm{f} 0$ are evident as significant differences in vocal tract length emerge.

The present study demonstrated significant positive moderate correlation between SSI3 scores and both jitter and shimmer of prolonged /a /vowel. This finding indicated that more severe stutterers exhibit more disturbed laryngeal functioning and weaker laryngeal and respiratory neuromuscular control than less severe stutterers. Contradictory to the present results, Hall and Yairi [5] showed that acoustic parameters were not correlated with stuttering severity. 
None of the acoustic parameters in the present study were significantly associated with Bloodstien grade in any of the speech samples. This could be explained by the fact that Bloodstien classification does not address the characteristics of speech problem, but relies on the awareness and secondary behaviors of stutterers.

Further studies are needed to confirm the effect of stuttering severity on acoustic parameters of voice and speech and to compare between pre- and post-treatment results using different treatment modalities.

\section{Conclusion}

The present study revealed significant differences in the acoustic parameters of voice and speech between Arabic-speaking stuttering children and normal children. Some of these acoustic parameters were significantly correlated with stuttering severity. Acoustic analysis can be used as simple, quick, and cheap tool for assessment of stuttering in children and might be a valuable addition to diagnostic set for assessment of stuttering severity.

\section{Abbreviations}

APA: Auditory perceptual assessment; BDMH: Brain Damaged Motorly Handicapped Children; CWS: Children who stutter; dB: Decibels; f0: Fundamental frequency; HZ: Hertz; SSI3: Stuttering Severity Instrument 3; VOT: Voice onset time.

\section{Acknowledgements}

We (all the authors) wish to thank all subjects who participated in this research.

\section{Authors' contributions}

All authors contributed to the study conception and design. ME was responsible for participant recruitment, data collection, and entry; shared in data interpretation; and wrote the main draft. OA was responsible for critical reviewing of the manuscript, shared in data interpretation, and submit the manuscript for publication. HB shared in reviewing and editing the manuscript. The authors approved the final version of the manuscript.

\section{Funding}

This research did not receive any specific grant from funding agencies in the public, commercial, or not-for-profit sectors.

\section{Availability of data and materials}

All datasets used are available.

\section{Declarations}

\section{Ethics approval and consent to participate}

The study protocol has been approved by the IRB committee of Mansoura Faculty of Medicine, Mansoura University, Egypt (MS.19.03.523). A written informed consent was signed by the parents of children participating in the study prior to the study.

\section{Consent for publication}

It was included in the written consent to participate in the study.

\section{Competing interests}

The authors declare that they have no competing interests.

\section{Author details}

${ }^{1}$ Phoniatric Unit, ORL Department, Damietta Specialized Hospital, Damietta, Egypt. ${ }^{2}$ Phoniatric Unit, ORL Department, Faculty of Medicine, Mansoura University, Mansoura 35516, Egypt.

Received: 5 October 2021 Accepted: 10 December 2021

Published online: 06 January 2022

\section{References}

1. Namasivayam AK, van Lieshout P (2011) Speech motor skill stuttering. J Mot Behav 43(6):477-489. https://doi.org/10.1080/00222895.2011.628347

2. Civier O, Tasko SM, Guenther FH (2010) Overreliance on auditory feedback may lead to sound/syllable repetitions: simulations of stuttering and fluency-inducing conditions with a neural model of speech production. J Fluen Disord 35(3):246-279. https://doi.org/10.1016/j.fludis.2010.05.002

3. Salihovic N, Junuzovic-Zunic L, Ibrahimagic A, Beganovic L (2009) Characteristics of voice in stuttering children. Acta Med Saliniana 38(2):67-75. https://doi.org/10.5457/ams.v38i2.55

4. Al-Tamimi F, Howell P (2020) Voice onset time and formant onset frequencies in Arabic stuttered speech. Clin Linguist Phon 35(6):493-508. https://doi.org/10.1080/02699206.2020.1786726

5. Hall KD, Yairi E (1992) Fundamental frequency, jitter, and shimmer in preschoolers who stutter. J Speech Lang Hear Res 35(5):1002-1008. https:// doi.org/10.1044/jshr.3505.1002

6. Boersma P, Weenink D ( 2013) Phonetic sciences Holanda: University of Amsterdam. Available from: http://www.fon.hum.uva.nl/praat/

7. Batalla FN, Márquez RG, González MBP, Laborda IG, Fernández MF Galán MM (2014) Acoustic voice analysis using the praat programme: comparative study with the Dr. speech programme. Acta Otorrinolaringol 65(3):170-176. https://doi.org/10.1016/j.otoeng.2014.05.007

8. Maryn Y (2017) Practical acoustics in clinical voice assessment: a praat primer. Perspect ASHA SIGs 2(SIG 3):14-32

9. Juste FS, Rondon S, Sassi FC, Ritto AP, Colalto CA, Andrade CRFD (2012) Acoustic analyses of diadochokinesis in fluent and stuttering children. Clinics 67:409-414

10. Hasseltine ES, Black SF, Corcoran TM, DiPalma DL, Dixon SE, Gooch AT et al (2016) Predicting stuttering severity ratings by timing and tallying dysfluencies using Praat software. Contemp Issues Commun Sci Disord 43(Spring):106-114

11. Rezai H, Tahmasebi N, Zamani P, Haghighizadeh MH, Afshani M, Tahzibi F, Heydari A (2017) Duration of stuttered syllables measured by "Computerized Scoring of the Stuttering Severity (CSSS)" and "Pratt". Iran Rehabil J 15(2):79-86

12. Bauerly KR, Paxton J (2017) Effects of emotion on the acoustic parameters in adults who stutter: an exploratory study. J Fluen Disord 54:35-49. https://doi.org/10.1159/000488758

13. Bauerly KR (2018) The effects of emotion on second formant frequency fluctuations in adults who stutter. Folia Phoniatr Logop 70(1):13-23. https://doi.org/10.1159/000488758

14. Bauerly KR, Jones RM, Miller C (2019) Effects of social stress on autonomic, behavioral, and acoustic parameters in adults who stutter. J Speech Lang Hear Res 62(7):2185-2202. https://doi.org/10.1044/2019 JSLHR-S-18-0241

15. Riley G (1994) Stuttering severity instrument for children and adults. ProEd, Austin

16. Bloodstein O (1993) Stuttering. The search for a cause and cure. (Allyn \& Bacon, Boston)

17. Paul B , David W (2017) Praat: doing phonetics by computer [Computer program]. Version 6.0. 21

18. Hammarberg B, Fritzell B, Gaufin J, Sundberg J, Wedin L (1980) Perceptual and acoustic correlates of abnormal voice qualities. Acta Otolaryngol 90(1-6):441-451. https://doi.org/10.3109/00016488009131746

19. Zraick RI, Wendel K, Smith-Olinde $L$ (2005) The effect of speaking task on perceptual judgment of the severity of dysphonic voice. J Voice 19(4):574-581. https://doi.org/10.1016/j.jvoice.2004.08.009

20. Roy N, Gouse M, Mauszycki SC, Merrill RM, Smith ME (2005) Task specificity in adductor spasmodic dysphonia versus muscle tension dysphonia. Laryngoscope 115(2):311-316. https://doi.org/10.1097/01.mlg.00001 54739.48314.ee 
21. Yiu E, Worrall L, Longland J, Mitchell C (2000) Analysing vocal quality of connected speech using Kay's computerized speech lab: a preliminary finding. Clin Linguist Phon 14(4):295-305. https://doi.org/10.1080/02699 200050023994

22. Bolfan-Stosic N, Prizl T (1998) Jitter and shimmer differences between pathological voices of school children. Fifth International Conference on Spoken Language Processing

23. Gharamaleki FF, Shahbodaghi MR, Jahan A, Jalayi S (2016) Investigation of acoustic characteristics of speech motor control in children who stutter and children who do not stutter. J Rehabil 17(3):232-243. https://doi.org/ 10.21859/jrehab-1703232

24. Wertzner HF, Schreiber S, Amaro L (2005) Analysis of fundamental frequency, jitter, shimmer and vocal intensity in children with phonological disorders. Br J Otorhinolaryngol 71(5):582-588. https://doi.org/10.1590/ S0034-72992005000500007

25. Baken RJ (1996) Clinical measurement of speech and voice. Singular Publishing Group, Inc, San Diego

26. Zocchi L, Estenne M, Johnston S, Del Ferro L, Ward ME et al (1990) Respiratory muscle incoordination in stuttering speech. Am Rev Respir Dis 141(6):1510-1515. https://doi.org/10.1164/ajrccm/141.6.1510

27. Schmitt LS, Cooper EB (1978) Fundamental frequencies in the oral reading behavior of stuttering and nonstuttering male children. J Commun Disord. https://doi.org/10.1016/0021-9924(78)90050-3

28. Cutler A (2005) Lexical stress. In:D. B. Pisoni \& R. E. Remez (eds.) The handbook of speech perception. Blackwell, Oxford. 264-289.

29. Fosnot S M, Jun S (1999) Prosodic characteristics in children with stuttering or autism during reading and imitation. Proceedings of the 14th international congress of phonetic sciences, 1925-1928

30. Wolfe V, Fitch J, Cornell R (1995) Acoustic prediction of severity in commonly occurring voice problems. J Speech Lang Hear Res 38(2):273-279. https://doi.org/10.1044/jshr.3802.273

31. Yavaş M (2011) Patterns of cluster reduction in the acquisition of \#sC onsets: are bilinguals different from monolinguals? Clin Linguist Phon 25(11-12):981-988. https://doi.org/10.3109/02699206.2011.616643

32. Abo-Ras YA, El-Maghraby R, Abdou RM (2013) The normative study of acoustic parameters in normal Egyptian children aged 4-12 years. Alex J Med 49(3):211-214

33. Toki El, Plachouras K, Tatsis G, Chronopoulos SK, Tafiadis D et al (2018) The design of a mobile system for voice e-assessment and vocal hygiene e-training. advances in intelligent systems and computing. Springer International Publishing, New York, pp 167-174

34. Fitch WT, Giedd J (1999) Morphology and development of the human vocal tract: a study using magnetic resonance imaging. J Acoust Soc Am 106(3):1511-1522. https://doi.org/10.1121/1.427148

\section{Publisher's Note}

Springer Nature remains neutral with regard to jurisdictional claims in published maps and institutional affiliations.

\section{Submit your manuscript to a SpringerOpen ${ }^{\circ}$ journal and benefit from:}

- Convenient online submission

- Rigorous peer review

- Open access: articles freely available online

- High visibility within the field

- Retaining the copyright to your article 